\title{
Research and implementation of high-availability model of information application system
}

\author{
Wang Shanshan \\ Officers College Of CAPF,Chengdu,610213,China \\ email:81792741@qq.com
}

\begin{abstract}
With the thorough application of information technology in the national social and economic development, the dependency of all walks of life on information application system increasingly increases. The demands on the high availability of modern information application system also are increasing. Thus, the selection of proper high availability model is the key to the successful design of information application system. This paper points out the design method of high availability of information application system and studies the implementations of check point model, process pairs model, recovery processing model, alternative treatment model and various high availability models of information application systems and also compares the implementation applicability of various models in information application system.
\end{abstract}

Keywords: Information application; high-availability; robustness; implementation model

\section{Introduction}

With the thorough application of information technology in the national social and economic development, the dependency of all walks of life on information system increasingly increases. The demands on the high availability of information system also are increasing. Many important core application systems (for example, government, communication, transportation, electric power and so on) even put forward $99.999 \%$ annual reliable operation rate, which means that the annual unplanned shutdown of the system shall be no more than 5 minutes. Usually, these systems with high demands on availability are called high-availability or high reliability system [1].

At present, scholars at home and abroad have carried out high-availability studies on information application system. However, most of them are specific analyses on the high-availability models of one or more specific information application systems. However, the overall and systematic research and analysis on the high-availability models of information application system are few and current studies also do not refer to the applicability of models. 
This paper points out the design method of high availability of information application system and studies the implementations of check point model, process pairs model, recovery processing model, alternative treatment model and various high availability models of information application systems and also compares the implementation applicability of various models in information application system.

\section{Design of the system's high-availability}

\subsection{Design objective}

The essence of information application system is to digitize the service management process and through various information systems generate new information resources, timely mastering all the information of various dynamic business for personnel at all levels and making decisions beneficial to reasonable resource allocation, aiming at achieve maximum economic or social benefits.

The design objective of high-availability of information application system is decided by its essence. Namely, under the condition of no manual intervention, redundancy technology of software and hardware is comprehensively applied to realize the automatic detection and correction of faults in the system operation to guarantee the continuous accessibility of key tasks or service [2].

\subsection{Design methods}

When the information application system operates, various temporary, permanent or discontinuous faults are the major influence factor of the system's high-availability. Thus, the design methods [2] [3] [4] to realize the system's high-availability include,

(1)Forecasting Fault

Forecasting Fault refers to using mathematical model and empirical formula to analyze and judge possible faults and harmful influences of the system in advance and change the occurrence conditions of faults to avoid faults.

(2)Avoiding and Removing Fault

Robust software and hardware technology is adopted to develop and realize the information system and at the same time and at the same time exact specification and perfect calibration test are needed to avoid and remove fault.

(3)Improving the Capacity of Tolerating Fault

Redundancy technology of software and hardware is adopted to avoid or remove the harmful influences of faults, namely, the system using redundant resources to recover the harmful influences of single-point fault and avoid the operation failure of the whole system.

Table 1 shows the statistics of the system faults of portal system $\mathrm{P}$ and directory system $\mathrm{D}$ of one unit in one year.

Table 1 Statistics table of the system faults of one unit in one year

\begin{tabular}{l|l|l|l} 
System & Platform & Application & External
\end{tabular}




\begin{tabular}{c|c|c|c|c} 
name & system fault & function fault & fault & fault \\
\hline Syste & 12 & 19 & 12 & 1 \\
m P & 13 & 16 & 13 & 2 \\
Syste & 13 & &
\end{tabular}

It can be seen from Table 1 that Method (1) and Method (2) can eliminate most system faults while it cannot guarantee that the system never has fault. Thus, Method (3) is adopted, which supposes that the system faults is sure to occur and coping strategies are established and redundancy technology of software and hardware is adopted to avoid or remove the harmful influences of faults. It is a more effective design method of the system's high availability in engineering practice. Figure 1 describes the redundancy design method to improve the tolerating fault capability of the system.

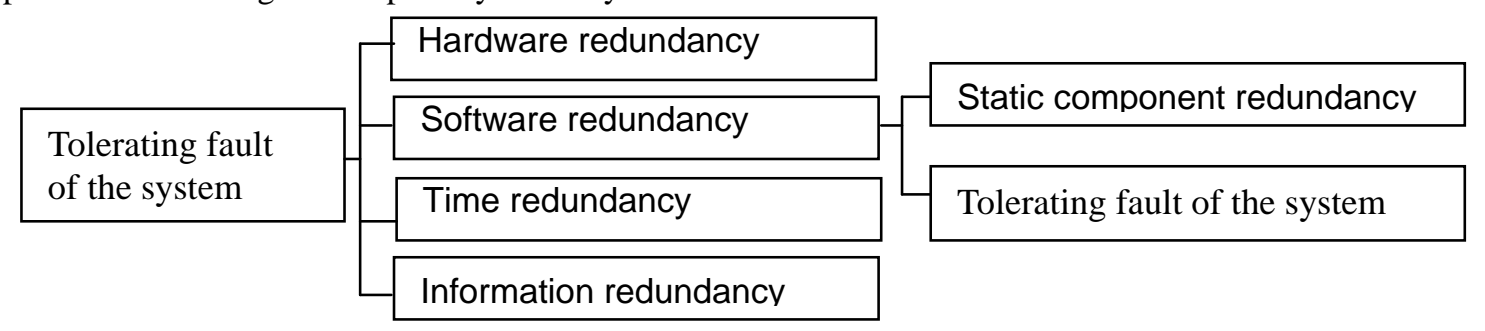

Fig.1. Common redundancy design method of the system's tolerating fault

Hardware redundancy The key module of hardware of the operation system adopts redundancy design, called N-Plexing design.

Software redundancy The key functional component of the system software adopts redundancy design. Software redundancy can be divided into static component redundancy and dynamic component redundancy. I $\mathrm{f}$ all the redundancy components are in active state, it is called static component redundancy, for example, the cluster model of software; if there is only one component is in active state while the others are in passive armed state, it is called dynamic component redundancy, for example, hot-standby model.

Time redundancy When the system has occasional faults, it will repeatedly implement the function module with fault and utilize time continuity to remove faults, for example, data retransmission mechanism of network link layer.

Information redundancy For important information about the system' storage, transmission and processing, data backup, 
additional checksum and error correcting code are used to guarantee the data accuracy.

\section{Implementation model of the system's high availability}

\subsection{Check point (CP) model}

On the basis of transaction concept, the system's state is saved when the transaction starts, called check point or snapshot. When there are faults in the transaction process, the system will roll back to the recently successful saved check point, including the deserted transaction with fault. The system restores to the consistency state and continues to operate and provide service. CP model is the most basic implementation model of the system's high availability and also is the base model of many implementation models.

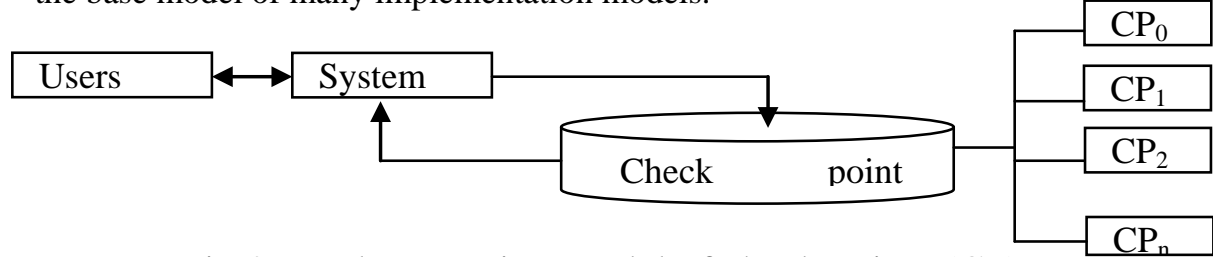

Fig.2. Implementation model of check points (CP)

\subsection{Process-pair (PP) model}

At the earliest, CP model was applied on stand-alone system. When the system has fault, the rolling-back speed to check points was slow. PP model is the improved CP model, aiming at speeding up the recovery process and decreasing the interruption time of the system service. The copy system of the main system is established and both share or synchronize the data of check points. The copy system determines the healthy state of the main system by receiving the heartbeat messages of the main system on time. When the copy system cannot receive the heartbeat messages of the main system and satisfy certain rules (for example, time-out), the copy system will automatically replace the main system to continue provide services.

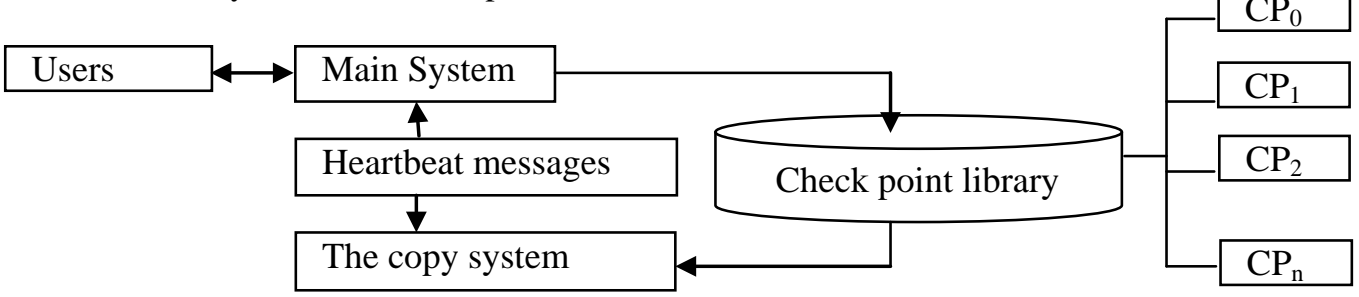

Fig.3. Implementation model of process-pair (PP) model

\subsection{Recovery processing (RP) model}


RP model is based on CP model. Specific to the key functions of the system, $\mathrm{N}$ mutually independent processing procedures with different algorithms $\{\mathrm{P} 0, \mathrm{P} 2, \ldots, \mathrm{PN}-1\}$ are established. Among them, one processing procedure is main processing procedure $\mathrm{P} 0$. Before $\mathrm{P} 0$, check point $\mathrm{CP} 0$ is established. After $\mathrm{P} 0$, result acceptance testing RAT0 is carried out. If the test fails, CP0 rolls back to $\mathrm{CP} 0$ and enters into processing procedure P1. After P1, result acceptance testing RAT1 is carried out. The processing function completes until the test

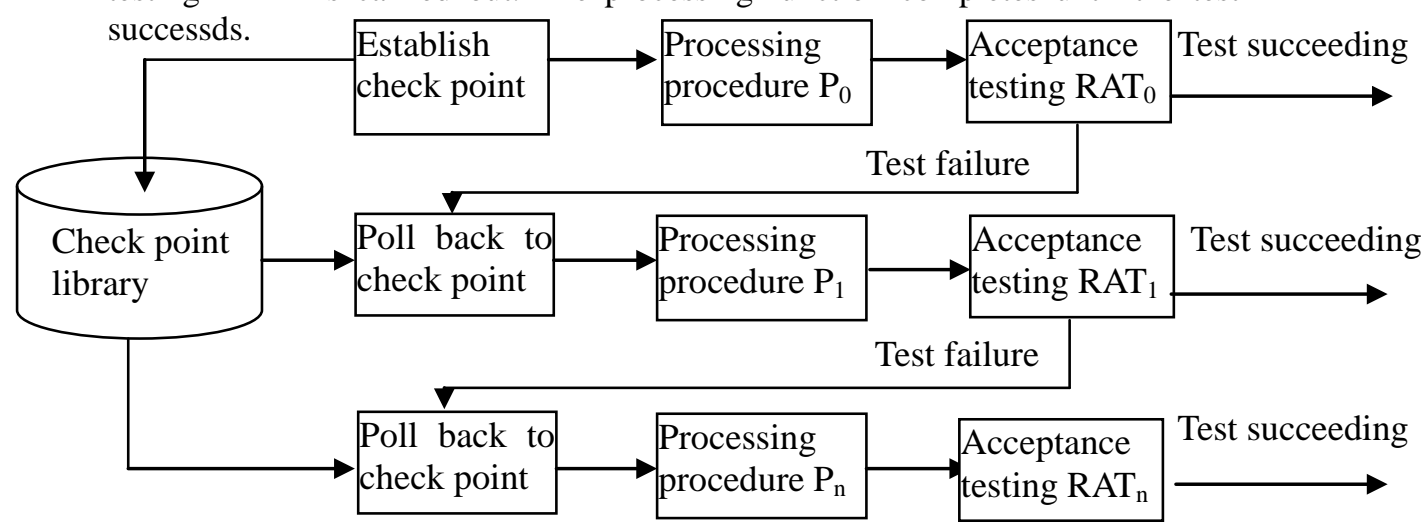

Fig.4. Implementation model of recovery processing (RP) model

\subsection{Alternative processing (AP) model}

Specific to the key function of transaction in the system, AP model establishes two mutually independent processing procedures with different algorithms, P1 and P0. Under general conditions, the algorithm of P0 processing procedure is complex and more accurate while the algorithm of $\mathrm{P} 1$ processing procedure is simple and more highly efficient. P1 can make receiving test on the results of P0 and also is the alternatively second processing procedure. P0 and P1 is controlled by decision-making process.

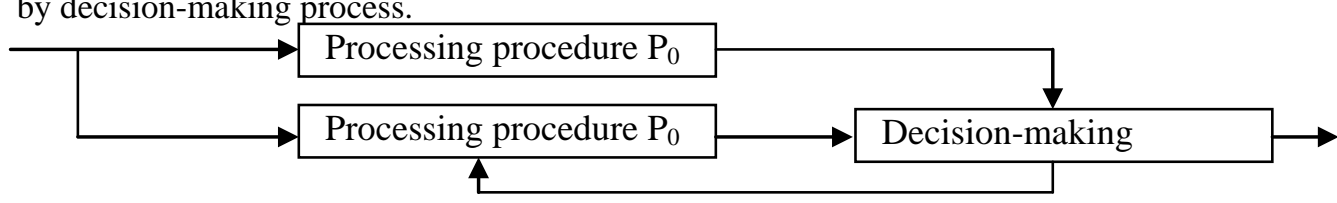

Fig. 5. Implementation model of alternative processing (AP) model

\subsection{Analysis on the model applicability}

The model applicability can be analyzed from applicable granularity, design difficulty, implementation cost, resource demands and other dimension for analysis. Applicable granularity includes system, module and process, respectively noted as $\mathrm{S}$ \{system\}, $\mathrm{M}\{$ module $\}$ and $\mathrm{P}\{$ process $\}$. Design difficulty, implementation cost and resource demands respectively noted as $\mathrm{H}$ 
(high),M (middle) and L (low). Analysis results of applicability of CP, PP, RP and AP models are shown as Table 2.

Table 2 Analysis results of the model applicability

\begin{tabular}{c|c|c|c|c} 
Applicability & $\begin{array}{c}\text { Applicable } \\
\text { granularity }\end{array}$ & $\begin{array}{c}\text { Design } \\
\text { difficulty }\end{array}$ & $\begin{array}{c}\text { Implementation } \\
\text { cost }\end{array}$ & $\begin{array}{c}\text { Resource } \\
\text { demands }\end{array}$ \\
\hline CP & S,M,P & M & $\mathrm{L}$ & $\mathrm{L}$ \\
PP & S,M & M & M & $\mathrm{H}$ \\
RP & M & H & H & H \\
AP & M & M & M & M
\end{tabular}

It can be seen from Table 2 that the applicable granularities of CP model and PP model are wide and their design difficulty, implementation cost and resource demands are not high; the applicable granularities of RP model and AP model are small while their design difficulty, implementation cost and resource demands are high. Thus, CP model and PP model have been widely applied in the current information application system while RP model and AP model are not frequent.

\section{Conclusion}

When selecting the implementation model with high availability, information application system shall fully consider the difference in applicable granularity, design difficulty, implementation cost and resource demands of models. According to the criticality of the system, annual reliable operation rate, information construction budget and other practical situations, the most applicable model shall be selected to construct the high-availability system. At the same time, with the development of virtualization, cloud computation and other technology, virtualization technology-based high-availability system occurs [5]. Compared with the traditional design, the system reliability, fault recovery time, resource use efficiency and other aspects have been improved, which shall be the new study direction of the future high availability technology.

\section{References}

[1]Trivedi K S, Vasireddy R, Trindade D, et al. Modeling high availability systems[C]//Proc. Pacific Rim Dependability Conference. 2006: 11-20.

[2] Jeske D R, Zhang X. Some successful approaches to software reliability modeling in industry[J]. Journal of Systems and Software, 2005, 74(1): 85-99.

[3] Gray J, Siewiorek D P. High-availability computer systems[J]. Computer, 1991, 24(9): 39-48.

[4] Christofferson M. Get High Availability Using Effective Fault Management[J]. Communication Systems Design, 2002, 8(7): 34-42.

[5] Clark C, Fraser K, Hand S, et al. Live migration of virtual machines[C]//Proceedings of the 2nd conference on Symposium on Networked Systems Design \& Implementation-Volume 2. USENIX Association, 2005: 273-286. 
[6]Liu Huan ,Shen Jiajun,Shi Guochuan. Discusses of High-availability technology of computer system [J].Computer and information technology. 2008(Z1).

[7]Zuo Ting,Wu Huijun. Analyis of the structure and principle of Linux high-availability system [J]. Journal of Jilin normal university (Natural science edition). 2007(04) .

[8]Sven Meth. Customizable modular control system [J]. Modern manufacturing. 2005(14) .

[9]Yao Jie,Xue Youwei. The principle of the high-availability cluster multiprocessing technology planning and allocation [J]. Electronic engineer. 2005(12). 